\title{
Proceso Generativo de Texturas Paramétricas Bioinspiradas: Modelo Metodológico de experimentación.
}

\author{
Generative Process of Bioinspired Parametric Textures: Methodological Model of \\ Experimentation.
}

\author{
Ever Patiño Mazo \\ Universidad Pontificia Bolivariana, Colombia \\ ever.patino@upb.edu.co \\ David Andrés Torreblanca Díaz \\ Universidad Pontificia Bolivariana, Colombia \\ david.torreblanca@upb.edu.co
}

\author{
Andrés Valencia-Escobar \\ Universidad Pontificia Bolivariana, Colombia \\ andres.valencia@upb.edu.co \\ Alejandro Zuleta Gil \\ Universidad Pontificia Bolivariana, Colombia \\ alejandro.zuleta@upb.edu.co
}

\begin{abstract}
This article presents the development of a bioinspired generative model based on principles of biological growth, which aim is to build of an on line open source textures repertoire that can be used in design and / or architecture projects. The project is developed in three phases: (i) characterization and abstraction of biological referents, (ii) definition of the transformations and (iii) fabrication of the repertory. This text is focused on the second phase, in addition to the assignation of the experiments using the Taguchi method. Finally, all the phases were validated by means of a case study.
\end{abstract}

Keywords: Sistemas generativos; Bioinspiración; Fabricación digital; Taguchi; Repertorio de texturas.

\section{Introducción}

La bioinspiración hace parte de las llamadas disciplinas de diseño biológicamente informado, a la que también pertenecen la biomimética y la biónica (A. louguina, et al., 2014). Esa gran sombrilla cobija las disciplinas que toman como referencia a la naturaleza para la solución de problemas humanos. Esta referencia puede enfocarse a la identificación, análisis y síntesis de principios o leyes de funcionamiento, materialización o configuración formal de origen natural, que de alguna manera brinden un apoyo al proyecto. De igual forma, se ha comprobado que si un objeto industrial se diseña a partir de la imitación de principios biológicos, tendrá mayor probabilidad de adquirir una serie de características que lo harán innovador y eficiente (Vincent, et. al., 2006; Jirapong, Krawczyk, \& Elnimeiri, 2002; Wen, et. al., 2008; Patiño, Arango \& Jaramillo, 2015).

De estas disciplinas de diseño biológicamente informado, la bioinspiración se encarga específicamente del análisis por analogía de una geometría natural particular; es decir, que hace parte del diseño por analogía, el cual estudia un dominio para transferir sus características por medio de la asociación a otro dominio que presenta problemas de diseño (Moreno D. et al., 2015). En este caso, el primer dominio son las superficies vegetales, las características hacen referencia a las propiedades geométricas y el segundo dominio son superficies y texturas de objetos, textiles y espacios.

Asimismo, un sistema generativo es un sistema computacional en el que se definen unos principios de formación y él es capaz de generar alternativas y variaciones de diseño alcanzando un nivel de complejidad incluso igual al de un sistema natural (Gürsel, 2012, p.208; Schumacher, 2008, p.6; Abdelmohsen,
2015, p.479). Para ello, se utilizan diferentes softwares que permiten la programación de los algoritmos, para posteriormente manipular las variables, con el fin de encontrar diferentes geometrías que respondan mejor a los requerimientos planteados. Posteriormente, las geometrías deben ser evaluadas y adaptadas a las condiciones iniciales del proyecto (Celani, 2008; Burry \& Maher, 2008).

El diseño generativo presenta diferentes características que lo convierten en una herramienta útil y práctica, entre ellas, que es un sistema que automatiza la etapa de búsqueda de morfológicas (Shea, Aish, \& Gourtovaia, 2005; Singh, \& Gu, 2012), permitiendo generar infinitas posibilidades de las formas iniciales a partir de la manipulación controlada de las variables (Szalapaj, 2014); permite una transición fácil a la manufactura digital; genera formas de alta complejidad que por otros medios serían muy difíciles de lograr diseñar, fabricar y comprender (McCormack, Dorin, \& Innocent, 2004; Menges, 2005; Chase, 2005; Oxman, 2006); y además, en el mismo proceso emergen formas que aumentan el tamaño del repertorio morfológico de los diseñadores (Hopf, 2009).

Los sistemas generativos que usan algoritmos basados en principios biológicos no son nuevos. Philip Galanter (2012) menciona, por ejemplo, al menos 12 sistemas generativos computacionales basados en procesos bioreferenciados que han sido utilizados en diferentes proyectos creativos, como: sistemas $L$, simuladores de caos, sistemas de reacción y difusión, vida artificial, sistemas aleatorios, simetrías y repeticiones, fractales, mapeo de datos y construcción combinada. A pesar de lo anterior, hay falencia de modelos generativos de código abierto, que puedan ser aplicados en el diseño de superficies y texturas para el diseño de productos y 
espacios, en los que se vinculen simultáneamente diferentes tipos de trasformaciones geométricas.

Es por ello, que en función de diseñar un repertorio de superficies y texturas bioinspiradas para profesionales de la Industria Culturales y Creativas, se origina el proyecto Repertorio de superficies y texturas bioinspiradas, a través de experimentaciones morfológicas con Tecnologías de Fabricación Digital. Con este proyecto se espera proveer un catálogo en código abierto, personalizable y en línea donde los diseñadores $y / 0$ arquitectos puedan extraer la mejor solución para un problema específico en el ámbito de las superficies y texturas de objetos, textiles y/o los espacios. En el proyecto se propone un modelo metodológico de generación morfológica, que presenta en total 6 tipos de transformaciones, cada una con dos niveles, que pueden utilizarse para variar la geometría de texturas tridimensionales sin perder sus propiedades morfológicas fundamentales.

En suma, este artículo tiene el objetivo de plantear un modelo de diseño generativo, que potencialice el tipo de transformaciones y disminuya el número de experimentos a partir de información estadística. Para esto, el texto se divide en tres partes: (i) descripción del modelo generativo propuesto, con la definición conceptual y geométrica de las transformaciones; (ii) selección del número y el tipo de experimentos a realizar utilizando la matriz ortogonal del método de Taguchi, y por último, (iii) ejemplificación de las fases del proyecto y del modelo generativo a partir de una caso de específico.

\section{Descripción del Modelo Generativo}

El modelo metodológico propuesto se compone de 6 tipos de transformaciones, cada una con dos niveles, organizadas en dos grupos (figura 1). El primer grupo son las transformaciones básicas; este agrupa 3 transformaciones: distancia, dislocación y rotación. El segundo grupo lleva por nombre transformaciones complejas y reúne: el crecimiento lineal, el crecimiento diferencial en dos direcciones y el crecimiento diferencial topológico. Ambos grupos se basan en principios biológicos y tienen un nivel "a" de transformación progresivo, y un nivel "b" de transformación aleatoria. A continuación se describen los dos grupos, definiendo conceptual y geométricamente las transformaciones.

\section{Transformación básica}

Este tipo de transformación está fundamentada, como se ve en la figura 1, en los procesos biológicos de ordenamiento. Específicamente, en las repeticiones y transformaciones geométricas que dejan el aspecto del objeto prácticamente igual, es decir las simetrías (Stewart \& Golobtsky, 1995, p.42). Las simetrías en la naturaleza tienen como principal objetivo el disminuir la información necesaria para construir, en el sentido en que necesita menos información genética un individuo con simetría bilateral, a un individuo en el que todas sus partes sean diferentes (Wagensberg, 2004). En el reino vegetal, en este caso, en las superficies que separan al individuo de su entorno, predominan la simetría radial y la de traslación. En la simetría radial hay un eje imaginario que rota alrededor de un centro, y en la de traslación las partes de mueven en una dirección a través de un eje. Esto ocasiona que la textura resultante se perciba visualmente ordenada, mientras se sigue una lógica de construcción equilibrada como efecto de partes consistentes y estables (Arbeláez \& Patiño, 2010).

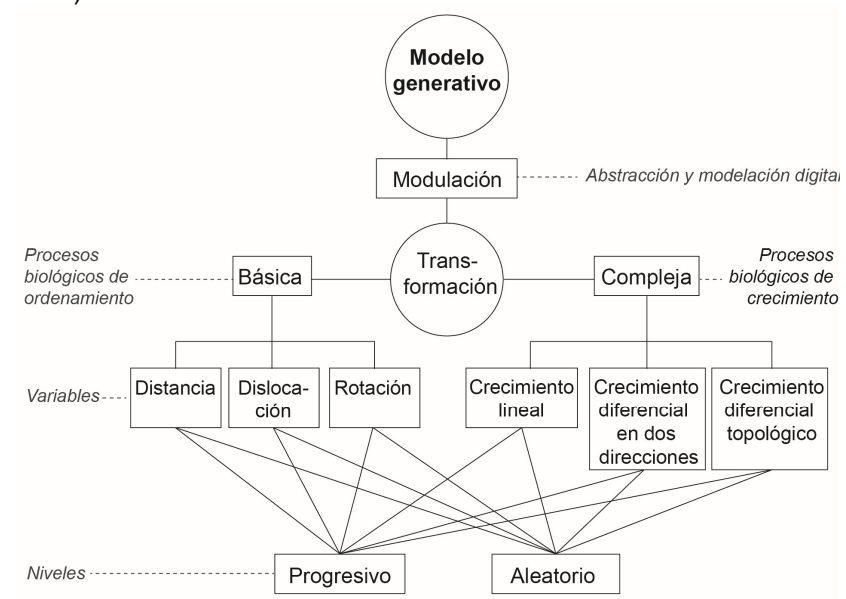

Figura 1: esquema del modelo generativo de experimentación con texturas (imagen de los autores).

Las tres variables que se determinaron fueron entonces, la distancia y la dislocación relacionadas con la simetría bilateral, y la rotación relacionada con la simetría radial. Igualmente, ya que la uniformidad y la precisión en las texturas naturales sólo son posibles en un entorno sin ningún cambio ambiental o perturbación, las variables tienen dos niveles: el nivel progresivo, y el nivel aleatorio. Ambos están fundamentados en el efecto ecofenotípico, que es, el efecto en la geometría que producen los fenómenos del entorno (Williams, 1984); en el nivel progresivo hay una transformación con unos valores periódicos y en el aleatorio se asignan aleatoriamente los valores en una franja específica.

En la tabla 1 se encuentran las convenciones que se utilizan en las descripciones matemáticas de las transformaciones; en la tabla 2 se describen matemáticamente las transformaciones para las tres variables con sus niveles; y para completar la explicación, de la figura 2 a la 4 se encuentran los esquemas gráficos que ejemplifican las transformaciones.

Tabla 1: Convenciones para las descripciones matemáticas.

\begin{tabular}{|cccc|}
\hline \multicolumn{2}{c}{ Descripción } & Descripción \\
\hline $\mathrm{XA}$ & Ancho del módulo & $\mathrm{C}$ & Altura del centro \\
\hline $\mathrm{XB}$ & Largo del módulo & $\mathrm{XX}$ & Ancho del formato \\
\hline $\mathrm{Y}$ & $\begin{array}{c}\text { Distancia entre } \\
\text { módulos }\end{array}$ & $\mathrm{P}$ & $\begin{array}{c}\text { Vertices y centro del } \\
\text { formato }\end{array}$ \\
\hline $\mathrm{F}$ & Fila & $\mathrm{n}$ & Sin transformación \\
\hline $\mathrm{Z}$ & Valor indeterminado & $*$ & $\begin{array}{c}\text { Atractor ubicado en } \\
\text { las esquinas o en el } \\
\text { centro }\end{array}$ \\
\hline$\alpha$ & Ángulo & $* *$ & $\begin{array}{c}\text { XA y XB mantienen la } \\
\text { proporción constante }\end{array}$ \\
\hline $\mathrm{H}$ & Altura del módulo & & \\
\hline
\end{tabular}


Tabla 2: Descripción matemática de las transformaciones básicas.

\begin{tabular}{|lcc|} 
& $\begin{array}{c}\text { Nivel a } \\
\text { (Progresivo) }\end{array}$ & $\begin{array}{c}\text { Nivel b } \\
\text { (Aleatorio) }\end{array}$ \\
\hline Distancia & $\begin{array}{c}\text { Atractor }{ }^{*}[\text { entre } Y=0 \\
\left.Y=X A(1.618)^{2}\right]\end{array}$ & $\begin{array}{c}\text { Entre } Y=0 \text { y } Y= \\
X A(1.618)^{2}\end{array}$ \\
\hline & En $F_{1}\left(Y_{1}=Z\right)$, & \\
en $F_{2}\left[Y_{2}=Y_{1}(1.1)\right]$ & \\
Dislocación & en $F_{3}\left[Y_{3}=Y_{1}(1.1)\right]$ & Entre $Y_{=} Y_{1}(1.1)$ y $Y=$ \\
& en $F_{4}\left[Y_{4}=Y_{1}(1.2)\right]$ & $Y_{1}(1.8)$ \\
& en $F_{5}\left[Y_{5}=Y_{1}(1.3)\right]$ & \\
& en $F_{6}\left[Y_{6}=Y_{1}(1.5)\right]$ y & \\
& en $F_{7}\left[Y_{7}=Y_{1}(1.8)\right]$ & \\
Rotación & $\alpha_{1}=10^{\circ}, \alpha_{2}=10^{\circ}$, & \\
& $\alpha_{3}=20^{\circ}, \alpha_{4}=30^{\circ}$, & \\
& $\alpha_{5}=50^{\circ}, \alpha_{6}=80^{\circ}$, & Entre $\alpha=0^{\circ}$ y $\alpha=360^{\circ}$ \\
& $\alpha_{7}=130^{\circ}, \alpha_{8}=210^{\circ}$, & \\
$\alpha_{9}=340^{\circ}$ & \\
\hline
\end{tabular}

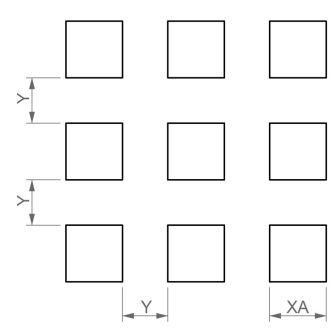

Figura 2: esquema gráfico de la transformación básica de distancia (imagen de los autores).

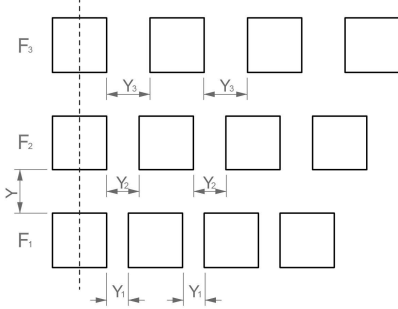

Figura 3: esquema gráfico de la transformación básica de dislocación (imagen de los autores).

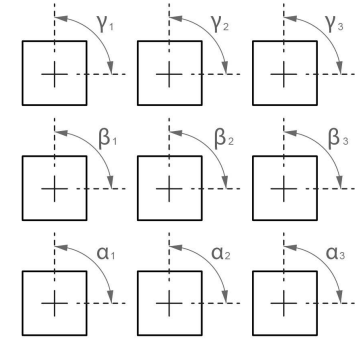

Figura 4: esquema gráfico de la transformación básica de rotación (imagen de los autores).

\section{Transformación compleja}

De manera similar, la transformación compleja está fundamentada en procesos biológicos de crecimiento, en especial principios que generan y transforman las morfologías. Teóricamente, la velocidad de crecimiento de todas las partes del individuo y el punto de inicio de los mismos, influencian considerablemente la geometría del objeto natural. Por ejemplo, una fruta como la naranja, crece del interior hasta al exterior, hinchando sus células de una manera más o menos homogénea, esto hace que la fruta adquiera una geometría esférica (Wagensberg, 2004). Para el modelo, se seleccionaron los principios de crecimiento lineal y crecimiento diferencial ya que pueden variar la forma sin cambiar de manera radical sus propiedades geométricas (ver figura 1).

El crecimiento lineal, parte de un punto, que en la configuración global del individuo coincide con el órgano reproductor, crece en una dirección manteniendo la velocidad constante (Arbeláez \& Patiño, 2010). El crecimiento diferencial en dos direcciones está relacionado con la teoría de las transformaciones de Thompson (2000), crece en un plano cartesiano y ejemplifica los cambios de velocidad de crecimiento en un mismo individuo. El crecimiento diferencial topológico, en su nivel progresivo, sigue el principio de crecer periódicamente más rápido en su centro que en su periferia, produciendo una forma tridimensional curva (Stevens, 1987). Y por último, el crecimiento diferencial topológico, en su nivel aleatorio, vincula las velocidades de crecimiento en unos vectores perpendiculares a la superficie original, ocasionando una superficie irregular curva.

El crecimiento lineal y el diferencial en dos direcciones exhiben nuevamente los niveles de progresión y aleatoriedad, con el objetivo de vincular el efecto ecofenotípico en su geometría. En la tabla 3 se describen matemáticamente las transformaciones complejas para las tres variables con sus niveles; y para complementar la descripción, de la figura 5 a la 8 se encuentran los esquemas gráficos que ejemplifican las transformaciones.

Tabla 3: Descripción matemática de las transformaciones complejas.

\begin{tabular}{|c|c|c|}
\hline & $\begin{array}{c}\text { Nivel a } \\
\text { (Progresivo) }\end{array}$ & $\begin{array}{c}\text { Nivel b } \\
\text { (Aleatorio) }\end{array}$ \\
\hline $\begin{array}{l}\text { Crecimiento } \\
\text { lineal }\end{array}$ & $\begin{array}{c}\text { Atractor [entre } \\
\mathrm{H}=\mathrm{H}(1) \mathrm{y} \\
\left.\mathrm{H}=\mathrm{H}(1.618)^{2}\right]\end{array}$ & $\begin{array}{c}\text { Entre } \mathrm{H}=\mathrm{H}(1) \text { y } \\
\mathrm{H}=\mathrm{H}(1.618)^{2}\end{array}$ \\
\hline $\begin{array}{l}\text { Crecimiento } \\
\text { diferencial } \\
\text { en dos } \\
\text { direcciones } \\
(\mathrm{Xa} \text { y } \mathrm{Xb})\end{array}$ & $\begin{array}{c}\text { Atractor [entre } \\
X A=X A(1) y \\
\left.X A=X A(1.618)^{2}\right]^{\star *}\end{array}$ & $\begin{array}{c}\text { Entre }^{* *} X A=X A(1) y \\
X A=X A(1.618)^{2}\end{array}$ \\
\hline $\begin{array}{l}\text { Crecimiento } \\
\text { diferencial } \\
\text { topológico }\end{array}$ & $\mathrm{C}=\mathrm{XX} / 2$ & Entre $\mathrm{P}=0$ y $\mathrm{P}=\mathrm{XX} / 2$ \\
\hline
\end{tabular}

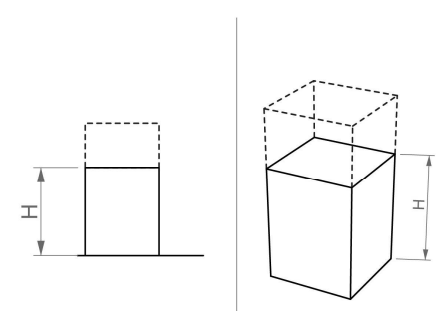

Figura 5: esquema gráfico de la transformación compleja de crecimiento lineal (imagen de los autores). 


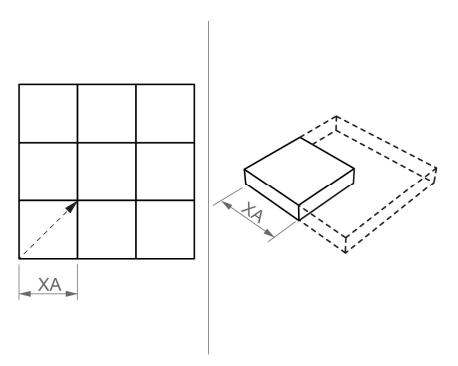

Figura 6: esquema gráfico de la transformación compleja de crecimiento diferencial en dos direcciones (imagen de los autores).

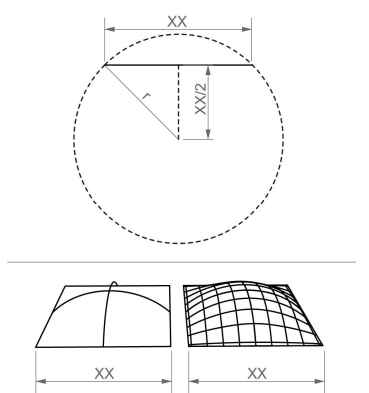

Figura 7: esquema gráfico de la transformación compleja de crecimiento diferencial topológico progresivo (imagen de los autores).

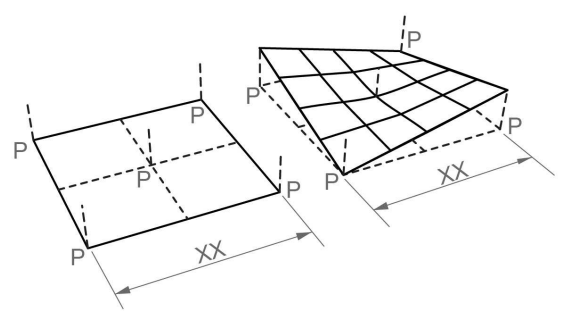

Figura 8: esquema gráfico de la transformación compleja de crecimiento diferencial topológico aleatorio (imagen de los autores).
Posteriormente, se determinaron tres criterios de evaluación, que permitieron, según la valoración más alta, seleccionar 24 especies vegetales que representaran cada subcategoría, para hacer la consecuente abstracción y representación digital utilizando el software CAD Rhinoceros 5.0. En la valoración participó un grupo de profesionales expertos en morfología, geometría, materiales y diseño, y se utilizó una escala de Likert de cinco ítems: nada, poca, neutral, bastante y mucha. A continuación se describen los tres criterios utilizados: (i) frecuencia, es el número de individuos que comparten propiedades morfológicas similares (Wagensberg, 2004); (ii) perspectiva de utilidad, es la posibilidad de construir puentes entre los modelos naturales y sus posibles aplicaciones (Baumeister, 2014); y atracción visual, es el placer estético producido por un objeto natural (Baumeister, 2014, p. 79)

Teniendo en cuenta que se definieron 6 tipos de transformaciones, y cada una de ellas tiene 2 niveles $\left(3^{2}+3^{2}\right)$, esto arrojaría 18 combinaciones posibles para cada una de las 24 especies, las cuales representan experimentos digitales independientes, para un total de 432 experimentos. Esta cantidad de experimentos hizo necesario recurrir al método de Taguchi, el cual está basado en técnicas estadísticas para determinar las mejores combinaciones en los experimentos, sobre todo en aquellos con múltiples variables (factores) y diferentes niveles, con el objetivo de disminuir la cantidad de experimentos a realizar (Wu \& Wu, 1997). Para este caso, al tener 3 transformaciones con dos niveles, se utilizó la matriz ortogonal $\mathbf{L}_{4}$ (ver tabla 4 y 5), permitiendo disminuir el número de experimentos de 9 a 4 por cada especie. Es decir, que no es necesario realizar 432 experimentos para establecer las tendencias de los resultados, porque el método elimina las combinaciones que no son relevantes.

Tabla 4: Matriz ortogonal $\mathrm{L}_{4}$ del método de Taguchi para las transformaciones básicas.

\begin{tabular}{|cccc|} 
& Distancia & Dislocación & Rotación \\
\hline $\mathbf{1}$ & $\mathrm{a}$ & $\mathrm{a}$ & $\mathrm{a}$ \\
\hline $\mathbf{2}$ & $\mathrm{a}$ & $\mathrm{b}$ & $\mathrm{b}$ \\
\hline $\mathbf{3}$ & $\mathrm{b}$ & $\mathrm{a}$ & $\mathrm{b}$ \\
\hline $\mathbf{4}$ & $\mathrm{b}$ & $\mathrm{b}$ & $\mathrm{a}$ \\
\hline
\end{tabular}

\section{Selección de Transformaciones por el Método de Taguchi}

En la primera fase del proyecto se registraron fotográficamente 225 especies biológicas, luego se generaron 12 grupos que se fueron agrupando y jerarquizando hasta llegar a sólo 6 categorías con sus respectivas subcategorías. Las categorías no responden a un ejercicio químico y/o biológico sino a una agrupación por características morfológicas más relevantes. Igualmente, no son excluyentes, si no por el contrario, es posible que una textura esté ubicada en una categoría pero gracias a la multifuncionalidad de las superficies biológicas, pueda incluir propiedades que respondan con menor relevancia visual a otra categoría. Las categorías fueron: geometrías básicas, reciprocas, relieves, ramificaciones, pliegues y textura visual.
Tabla 5: Matriz ortogonal $\mathrm{L}_{4}$ del método de Taguchi para las transformaciones complejas.

\begin{tabular}{|c|c|c|c|}
\hline & $\begin{array}{l}\text { Crecimiento } \\
\text { lineal }\end{array}$ & $\begin{array}{l}\text { Crecimiento } \\
\text { diferencial } \\
\text { (dos } \\
\text { direcciones) }\end{array}$ & $\begin{array}{l}\text { Crecimiento } \\
\text { diferencial } \\
\text { topológico }\end{array}$ \\
\hline 1 & a & a & a \\
\hline 2 & $a$ & $b$ & $b$ \\
\hline 3 & $b$ & $a$ & $b$ \\
\hline 4 & $b$ & $b$ & $a$ \\
\hline
\end{tabular}

Igualmente, de las 24 especies seleccionadas que daban respuesta a la subcategoría, se eligieron con los mismos criterios, 12 especies con la valoración más alta independiente de la subcategoría. La nueva selección y el uso del método de Taguchi hicieron que se disminuyeran a 96 el número de experimentos. 
Por otro parte, la geometría de las modelaciones digitales de las especies seleccionados limitan el tipo de transformaciones que se les puede aplicar. Por esto, se desarrollaron dos listas de chequeo que permitieron establecer, dependiendo de su geometría que transformaciones de pueden aplicar (ver tabla 6 y 7). En la figura 9 se puede ver una síntesis del proceso, donde se incluye una fotografía de la especie, la modelación digital, y las transformaciones. A continuación se explicará la aplicación de las transformaciones a partir de un estudio de caso.

Tabla 6: Lista de chequeo para seleccionar el conjunto de transformaciones dependiendo del tipo de modulación.

\begin{tabular}{|ccc|}
\hline & $\begin{array}{c}\text { Transformación } \\
\text { básica }\end{array}$ & $\begin{array}{c}\text { Transformación } \\
\text { compleja }\end{array}$ \\
\hline $\begin{array}{c}\text { Modulación } \\
\text { cerrada }\end{array}$ & $\mathrm{x}$ \\
\hline Modulación abierta & $\mathrm{x}$ & $\mathrm{x}$ \\
\hline
\end{tabular}

Tabla 7: Lista de chequeo para seleccionar el tipo de transformación compleja dependiendo de las propiedades geométricas del módulo. Un módulo unidimensional, es una geometría en el plano $\mathrm{XY}$, donde la textura es dada por los cambios en el pigmento; el módulo tridimensional es una geometría donde el largo, el ancho y el alto son proporcionalmente similares; y por último, el módulo plano con relieve, es una geometría con proporciones predominantes en el plano XY pero que exhibe un relieve en el eje $Z$.

\begin{tabular}{|cccc|}
\hline & $\begin{array}{c}\text { Crecimiento } \\
\text { lineal }\end{array}$ & $\begin{array}{c}\text { Crecimiento } \\
\text { diferencial } \\
\text { (dos } \\
\text { direcciones) }\end{array}$ & $\begin{array}{c}\text { Crecimiento } \\
\text { diferencial } \\
\text { topológico }\end{array}$ \\
\hline $\begin{array}{c}\text { Módulo } \\
\text { unidimensional }\end{array}$ & $\mathrm{x}$ & $\mathrm{x}$ & $\mathrm{x}$ \\
\hline $\begin{array}{c}\text { Módulo } \\
\text { tridimensional }\end{array}$ & $\mathrm{x}$ & $\mathrm{x}$ \\
\hline $\begin{array}{c}\text { Módulo plano } \\
\text { con relieve }\end{array}$ & $\mathrm{x}$ \\
\hline
\end{tabular}

\section{Aplicación del modelo}

Como se dijo anteriormente, y con el objetivo de aumentar la comprensión del modelo generativo propuesto, se ejemplificarán todas las fases del proyecto con una de las especies vegetales estudiadas (ver figura 9h). El proyecto del que hace parte el modelo, consta de 3 fases: caracterización y abstracción de modelos biológicos, experimentación morfológica digital y realización del repertorio.

En la primera fase, después de seleccionar las 12 especies, los modelos biológicos fueron abstraídos mediante un análisis visual digital empleando herramientas $C A D$ a partir de: puntos de transición, contornos, simetrías, secciones transversales y patrones básicos como líneas rectas, secciones de círculo, circunferencias, elipses y/o parábolas. En la figura 10, se puede ver el proceso de caracterización y abstracción de la piña de la especie vegetal Pinus pinea, la cual siguió el siguiente proceso utilizando el software Rhinoceros: a. Dibujo de ejes constructivos de la configuración global de la especie. Se trazaron círculos sobre la fotografía.

b. Selección de uno de los módulos y dibujo de los círculos constructivos de éste.

c. A partir de principios organizativos -simetría y proporción- se redibuja nuevamente la vista de planta y el corte del módulo.

d. Se modela tridimensionalmente la superficie del módulo, haciendo uso de las vistas dibujadas.

e. Repetición bidimensional del módulo dibujado.

f. Textura de la repetición tridimensional del módulo.

Para la segunda fase, se definieron las transformaciones básicas y complejas y se tradujeron en lenguaje de programación del plug-in Grasshopper de Rhinoceros. Para el caso específico de la textura de la Pinus pinea, se utilizó la lista de chequeo de la tabla 6 para asignar el tipo de transformación: básica y/o compleja. Como la modulación de esta textura es cerrada, es decir, que no tiene espacios vacíos o superficies planas entre los módulos, se determina que la transformación básica no es necesaria, ya que afectaría las propiedades geométricas del módulo. Es por esto que en la figura $9 \mathrm{~h}$ en la columna de transformación básica se le asigna la letra "n", que representa que no tiene transformación de este tipo.

De igual manera, se utilizó la lista de chequeo de la tabla 7 para seleccionar las transformaciones complejas. Al ser el módulo, una geometría cuyas dimensiones predominan en el plano $X Y$ y exhibe un relieve no tan predominante, se le asignan las transformaciones del módulo plano con relieve, es decir: Crecimiento diferencial en dos direcciones y crecimiento diferencial topológico. En la figura 11 se puede ver la definición del algoritmo en la interface de Grasshopper, de la transformación "nab" (ver figura 9h). La "n" representa que el crecimiento lineal no se hace, la "a" que el crecimiento diferencial en dos direcciones es de nivel progresivo, y la "b" que el crecimiento diferencial topológico es de nivel aleatorio.

De igual manera, en la figura 9 se muestran todas las transformaciones asignadas, a manera de código, para cada una de las texturas biológicas; como se dijo anteriormente, si el módulo presenta repetición cerrada no se le realiza transformación básica (ver tabla 6) y se le asigna una "n". Por otro lado, para las transformaciones complejas se tiene el código del tipo de experimento que se debe hacer según el método Taguchi.

Para terminar, en la tercera fase se utilizaron tecnologías aditivas y sustractivas para fabricar digitalmente las texturas abstraídas y las texturas transformadas. En las figuras 12a y $12 \mathrm{~b}$ se puede ver el proceso de corte láser de la textura sin transformaciones; en las figuras $12 \mathrm{c}, 12 \mathrm{~d}$ y $12 \mathrm{e}$ se observa el fresado CNC de la transformación de la textura; y en las figuras $12 \mathrm{f}, 12 \mathrm{~g}$ y $12 \mathrm{~h}$ se puede ver la impresión 3D de alta precisión por lámpara U.V, con resina epóxica termosensible. 


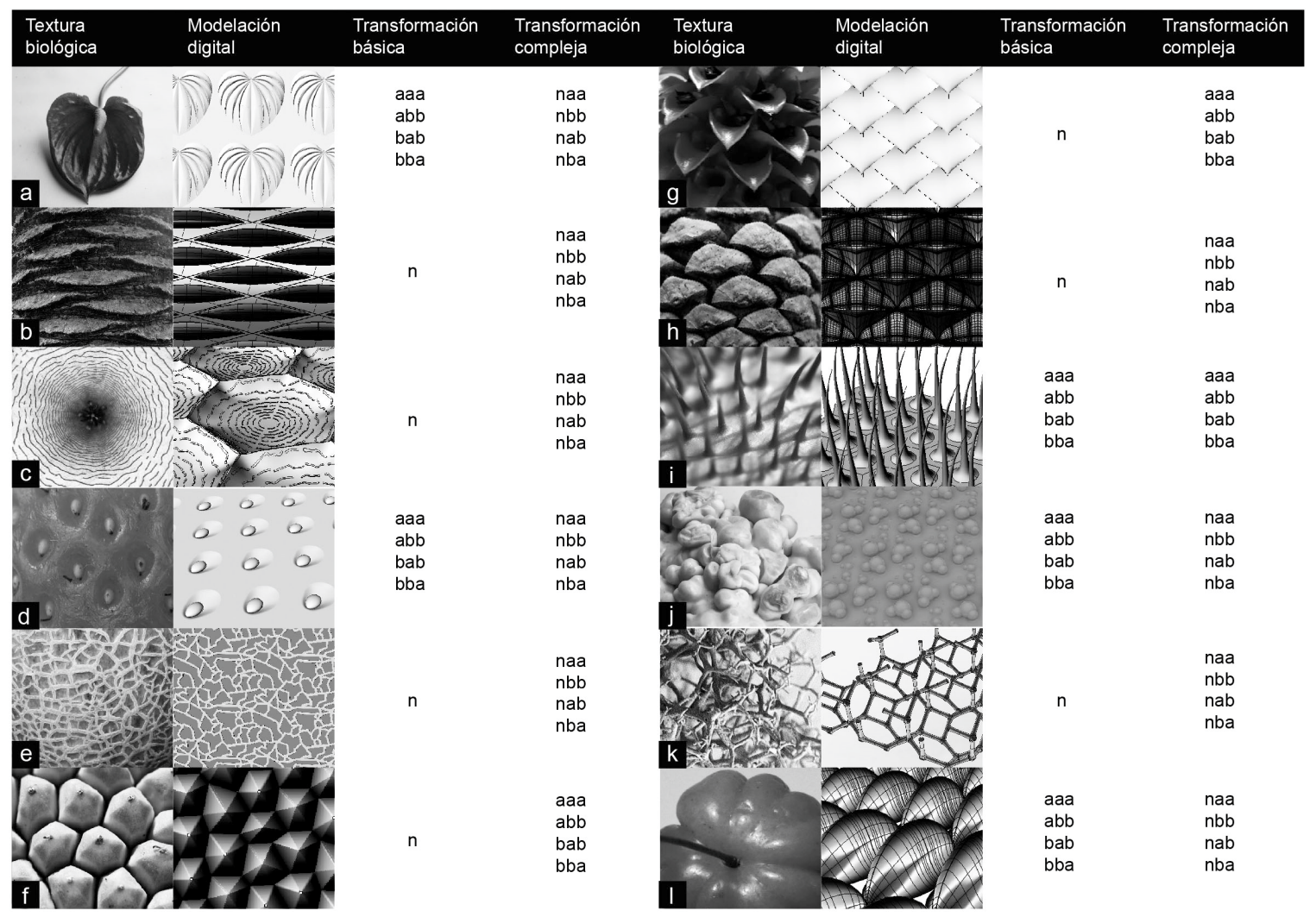

Figura 9: síntesis del proceso con las transformaciones asignadas (fotos e imágenes de Silvia Gallego, Carolina Cardona y Semillero de investigación MORFOlab).
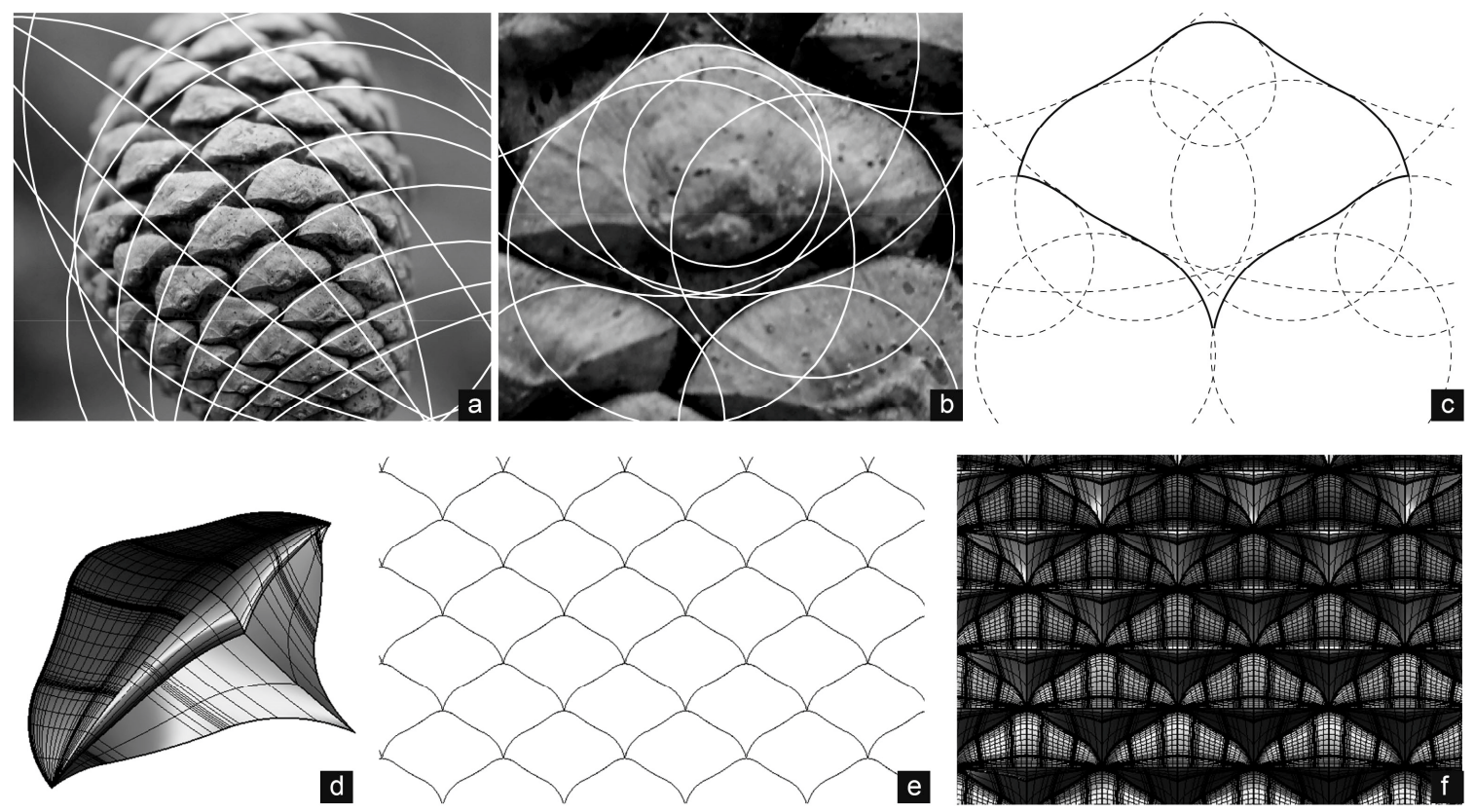

Figura 10: proceso de caracterización y abstracción de la piña de la Pinus pinea (fotografías de Pablo Montes, modelación digital de Julian Villa del semillero de investigación MORFOlab. 

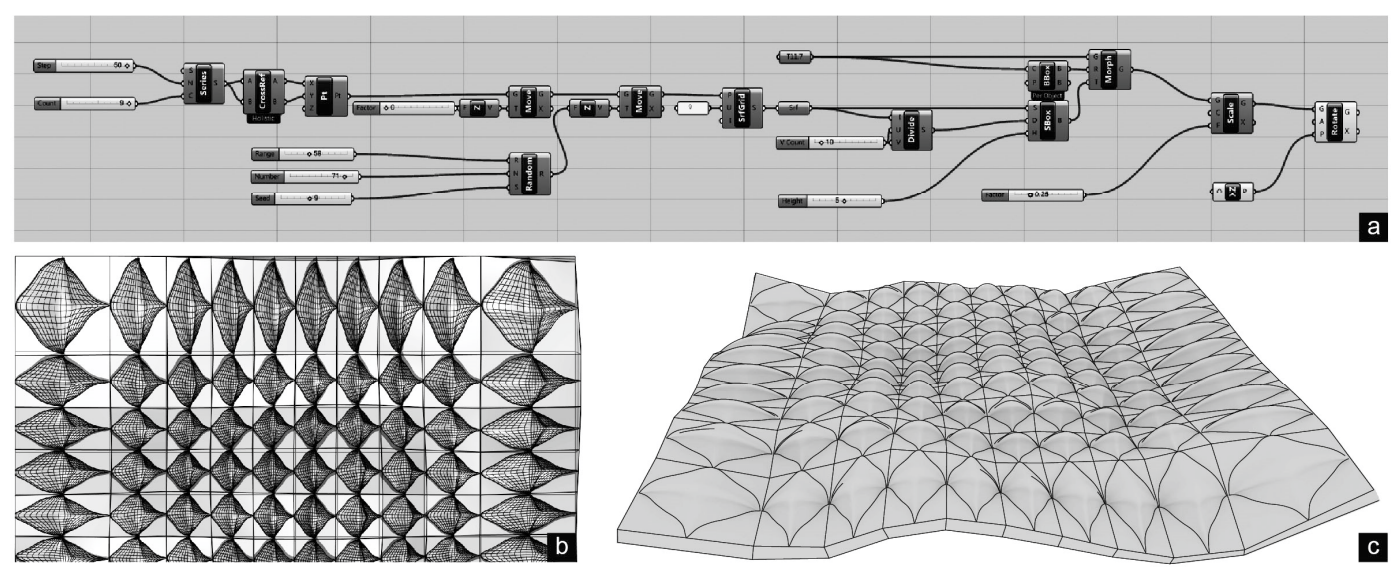

Figura 11: Transformación "nab" de la textura de la Pinus pinea desarrollada por el pasante de doctorado Byron Villamil de la Universidad Complutense de Madrid. La figura 11a es la definición de grasshopper, y las figuras 11b y 11c muestran la transformación en vista de planta y en perspectiva.

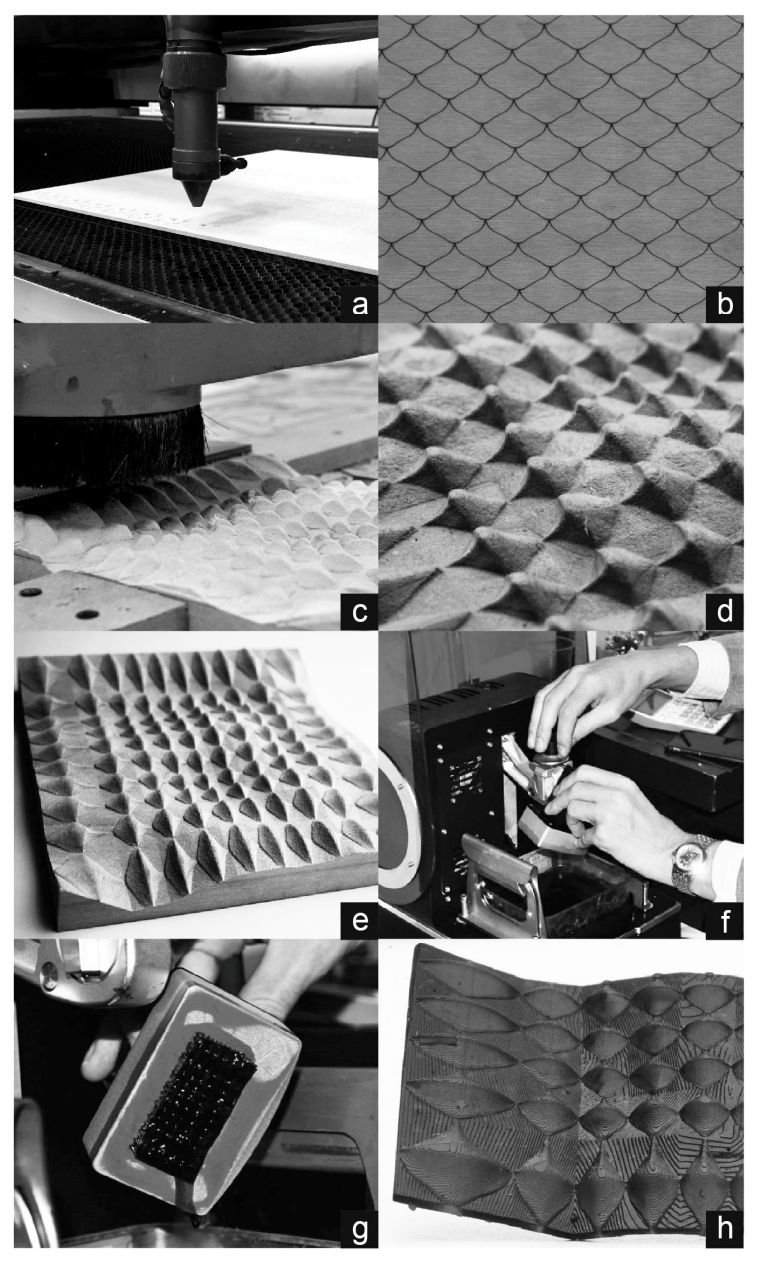

Figura 12: Fabricación de la textura de la Pinus pinea. Fotografías de Silvia Gallego, Carolina Cardona y David Torreblanca.

\section{Conclusiones}

Es importante mencionar que la totalidad del repertorio aún está en construcción, ya que en el proyecto actualmente se está ejecutando la tercera etapa. Pero del modelo generativo propuesto se puede concluir lo siguiente:

- La referenciación de las especies vegetales y el seguimiento de principios de crecimiento biológico en el diseño generativo, aunque ya ha aplicado de diferentes maneras, sigue arrojando resultados novedosos y llamativos a nivel morfológico.

- $\quad$ El uso del método de Taguchi dentro del modelo permitió aumentar la validez interna del experimento en la medida que se controlan todas las variables y éstas se pueden analizar eventualmente por separado. Es decir, que al evaluar los resultados de los experimentos se podrían analizar las relaciones de los tipos de transformaciones con la frecuencia, la perspectiva de utilidad y la atracción visual.

- Igualmente, la operacionalización de las variables de transformación, posibilita el control del proceso y de los resultados, aumentando la validez interna y por consiguiente, facilitando la replicabilidad del modelo.

- Por otro lado, aunque la metodología está planteada para ser utilizada en superficies y texturas, la estructura del modelo puede ser adaptada a otro tipo de procesos, favoreciendo la validez externa del proyecto.

- Por último, la utilidad del modelo y del repertorio sólo podrá ser comprobada al final del proyecto, en el momento en que se apliquen las texturas y se evalúen los resultados.

\section{Agradecimientos}

Damos las gracias al Semillero de investigación MORFOlab de la Universidad Pontificia Bolivariana, por las actividades de acompañamiento desarrolladas, y a la empresa Rhinoceros por apoyar la fabricación del repertorio. 
SIGraDi 2017, XXI Congreso de la Sociedad Ibero-americana de Gráfica Digital 22 - 24 Noviembre, 2017 - Concepción, Chile.

\section{Referencias}

Abdelmohsen, S. (2015) An Inquiry into Designing in Context using Generative Systems. International Journal of Architectural Computing. Vol 12. Nº 4. 477-494.

Arbeláez, E., \& Patiño, E. (2010). Generación y transformación de la forma. Medellín: UPB.

Baumeister, D. (2014). Biomimicry Resource Handbook: A Seed Bank of Best Practices. Biomimicry 3.8.

Bueno, E. (2010). Algorithmic form generation of a Radiolarian Pavilion. International journal of architectural computing. Issue 04, volumen 07. Pág. 677-688.

Burry, J. \& Maher, A. (2008). The Other Mathematical Bridge. Nexus Network Journal of Architecture and Mathematics. Vol. 10. $N^{\circ} 1.179-194$.

Celani, G. (2008). Enseñando diseño generativo: una experiencia didáctica. XII Congreso Sigradi, Cuba. Diciembre, 1-4.

Chase, S. C. (2005). Generative design tools for novice designers: Issues for selection. Automation in Construction, 14(6), 689-698.

Galanter, P. (2012). Computational Aesthetic Evaluation: steps towards machine creativity. SIGGRAPH 2012, Los Angeles, California. Agosto, 1-162.

Gönenç, A., Arslan, S. (2013). Computational Models in Architecture: Understanding Multi- Dimensionality and Mapping. Nexus Network Journal of Architecture and Mathematics. Vol. 15. №2. 349-362.

Gürsel, I. (2012). Creative Design Exploration by Parametric Generative Systems in Architecture. Metu Journal of the Faculty of Architecture. N²9. 207-224.

Hopf, A. (2009, September). Renaissance 2.0-Expanding the Morphologic Repertoire in Design. In 24th Cumulus Conference (Vol. 24, No. 09, pp. 78-85).

louguina, A., Dawson, J.W., Hallgrimsson, B., Smart, G. (2014). Biologically informed disciplines: a comparative analysis of bionics, biomimetics, biomimicry, and bio-inspiration among others. Int. J. of Design \& Nature and Ecodynamics. Vol. 9, No. 3. 197-205.

Jirapong, K., Krawczyk, R. J., \& Elnimeiri, M. (2002). Natural Forms as Virtual Architectures; en: Proceedings of the $20^{\text {th }}$ Conference on Education in Computer Aided Architectural Design in Europe, 14. Warsaw, 2002.

McCormack, J., Dorin, A., \& Innocent, T. (2004). Generative design: a paradigm for design research. Proceedings of Futureground, Design Research Society, Melbourne.
Menges, A. (2005). Pluripotent Components and Polymorphous Systems: An Alternative Approach to Parametric Design. AA Files, (52), 63-74.

Moreno, D. Blessing, L. Yang, M. Wood, K. (2015). The potential of design-by-analogy methods to support product, service and product service systems idea generation. International Conference on Engineering Design, Iced15 27-30 July 2015, Politecnico Di Milano, Italy.

Oxman, R. (2006). Theory and design in the first digital age. Design Studies, 27(3), 229-265.

Patiño, E. Arango, M. Jaramillo, J. (2015). Biomimética o la traducción de los fenómenos biológicos al diseño. Iconofacto. Vol. 11. Número 16. Enero - Junio. $201-212$.

Schumacher, P. (2008). Preface: Autopoietic Elegance. En: Research Institute for Experimental Architecture (Ed.), MORPHE MRGD (pp. 6-8). New York, E.E.U.U: Springer Wien New York.

Shea, K., Aish, R., \& Gourtovaia, M. (2005). Towards integrated performance-driven generative design tools. Automation in Construction, 14(2), 253-264.

Stevens, P. (1987). Patrones y pautas en la naturaleza. Barcelona: Salvat Editores, S.A.

Steward, I., \& Golubitsky, M. (1995). ¿Es Dios geómetra?: las simetrías de la naturaleza. Barcelona: Crítica.

Szalapaj, P. (2014). Contemporary architecture and the digital design process. Routledge.

Thompson, D. (2000). Sobre el crecimiento y la forma. Madrid, España: Cambridge University Press

Vincent, J. F., Bogatyreva, O. A., Bogatyrev, N. R., Bowyer, A., \& Pahl, A. K. (2006). Biomimetics: its practice and theory. Journal of the Royal Society Interface, 3(9), 471-482.

Wagensberg, J. (2004). La rebelión de las formas. O cómo perseverar cuando la incertidumbre aprieta. Barcelona: Tusquets Editores.

Wen, H. I., Zhang, S. J., Hapeshi, K., \& Wang, X. F. (2008). An innovative methodology of product design from nature. Journal of Bionic Engineering, 5(1), 75-84.

Williams, C. T., \& Williams, H. C. (1984). Los orígenes de la forma/Origins of form (No. 7.01). Gustavo Gili.

Wu, Y., \& Wu, A. (1997). Diseño robusto utilizando los métodos Taguchi. Ediciones Díaz de Santos. 